\title{
Injury of the Arcuate Fasciculus in the Nondominant Hemisphere by Subfalcine Herniation in Patients with Intracerebral Hemorrhage : Two Case Reports and Literature Review
}

\author{
Sung Ho Jang, M.D., ${ }^{1}$ Seong Ho Kim, M.D., ${ }^{2}$ Min Cheol Chang, M.D. ${ }^{3}$ \\ Departments of Physical Medicine and Rehabilitation, ${ }^{1}$ Neurosurgery, ${ }^{2}$ College of Medicine, Yeungnam University, Daegu, Korea \\ Department of Physical Medicine and Rehabilitation, ${ }^{3}$ Asan Medical Center, University of Ulsan College of Medicine, Seoul, Korea
}

\begin{abstract}
Using diffusion tensor tractography (DTT), we demonstrated injury of the arcuate fasciculus (AF) in the nondominant hemisphere in two patients who showed subfalcine herniation after intracerebral hemorrhage (ICH) in the dominant hemisphere. Two patients (patient 1 and patient 2) with ICH and six age-matched control patients who have $\mathrm{ICH}$ on the left corona radiata and basal ganglia without subfalcine herniation were recruited for this study. DTT was performed at one month after onset in patient 1 and patient 2. AFs of both hemispheres in both patients were disrupted between Wernicke's and Broca's areas. The fractional anisotropy value and tract numbers of the right AFs in both patients were found to be more than two standard deviations lower than those of control patients. In contrast, the apparent diffusion coefficient value was more than two standard deviations higher than those of control patients. Using the configuration and parameters of DTT, we confirmed injury of the AF in the nondominant hemisphere in two patients with subfalcine herniation following ICH in the dominant hemisphere. Therefore, DTT would be a useful tool for detection of underlying injury of the $\mathrm{AF}$ in the nondominant hemisphere in patients with subfalcine herniation.
\end{abstract}

Key Words : Subfalcine herniation · Arcuate fasciculus · Nondominant hemisphere · Diffusion tensor tractography · Broca's aphasia.

\section{INTRODUCTION}

Subfalcine herniation, the most common form of brain herniation, is characterized by lateral displacement of the brain beneath the falx cerebri due to increased intracranial pressure resulting from cerebral hemorrhage, traumatic brain injury, or brain tumor ${ }^{1)}$. It can cause injury to neural tracts directly by compressing the contralesional white matter or by compressing branches of the cerebral artery and secondarily inducing ischemic infarct ${ }^{4)}$. Injury to the neural tract caused by subfalcine herniation can accompany various neurological sequelae ${ }^{9)}$. Therefore, thorough evaluation of the state of the neural tracts in patients with subfalcine herniation is necessary for determination of the causes of neurological deficits and for establishment of specific rehabilitation strategies. However, injury of neural tracts by subfalcine herniation has not been clearly elucidated ${ }^{9)}$.

The arcuate fasciculus (AF) is the neural tract that plays an im- portant role in conduction of language processing between the two major speech centers of Broca's and Wernicke's areas ${ }^{5)}$. Therefore, injury to the AF can result in several language dysfunctions, including conduction aphasia, Broca's aphasia, apraxia of speech, and anomia ${ }^{3,5)}$. Intra-operative mapping techniques, conventional computed tomography (CT), and magnetic resonance imaging (MRI) have been used in examination of the state of the AF in patients with language dysfunction ${ }^{6,16,18)}$. However, these evaluation methods have significant limitation in that they do not have the capacity for visualization of the AF. By contrast, diffusion tensor tractography (DTT), which is derived from diffusion tensor imaging (DTI), can visualize the architecture and integrity of the AF in three dimensions ${ }^{12,13)}$. Therefore, use of DTT for detection of injury of the AF in stroke patients with language dysfunction is increasing ${ }^{10,12,13)}$. However, little is known about injury of the AF caused by subfalcine herniation.

In this study, using DTT, we report in injury of the AF in the

- Received : September 19, 2014 • Revised : February 6, 2015 • Accepted : February 10, 2015

- Address for reprints : Min Cheol Chang, M.D.

Department of Physical Medicine and Rehabilitation, Asan Medical Center, University of Ulsan College of Medicine, 88 Olympic-ro 43-gil, Songpa-gu, Seoul 05505, Korea Tel : +82-2-3010-3800, Fax : +82-2-3010-6964, E-mail : wheel633@hanmail.net

- This is an Open Access article distributed under the terms of the Creative Commons Attribution Non-Commercial License (http://creativecommons.org/licenses/by-nc/3.0) which permits unrestricted non-commercial use, distribution, and reproduction in any medium, provided the original work is properly cited. 
nondominant hemisphere by subfalcine herniation in patients following intracerebral hemorrhage (ICH) in the dominant hemisphere.

\section{CASE REPORT}

\section{Case presentation}

Two ICH patients with subfalcine herniation (patient 1 and patient 2) and six right-handed age-matched control patients who have ICH on the left corona radiata and basal ganglia without subfalcine herniation (three men; mean age, 44.0 years; range, 41-49 years) were recruited for this study. All subjects received speech therapy ( 5 days per week, Monday through Friday : 30 minutes/day) consecutively after transfer to the department of rehabilitation (around 2 weeks after onset) until 3 months after onset. All subjects provided informed consent for participation in the study. The study was approved by the Institutional Review Board of a university hospital.

Patient 1 was a 41-year-old right handed male who underwent craniotomy and hematoma removal for an $\mathrm{ICH}$ in the left corona radiata and basal ganglia in the neurosurgery department of a university hospital. Brain CT performed at onset revealed an $\mathrm{ICH}$ in the left corona radiata and basal ganglia, and a subfalcine herniation (7.0 mm left-to-right midline shift) (Fig. 1A). Brain MRI performed at one month after onset revealed a leukomalatic lesion in the same region (Fig. 1A). The Korean-Western Aphasia Battery (K-WAB) ${ }^{11)}$ was used for assessment of the patient's language function. At one month after onset, he showed global aphasia (aphasia quotient : 4 , fluency : 1 , comprehension : 38, repetition : 0 , naming : 0 ). However, at three months after onset, his K-WAB scores had improved, and the aphasia type was compatible with Broca's aphasia (aphasia quotient : 39, fluency : 6.3 , comprehension : 137 , repetition : 35 , naming : 42).

Patient 2 was a 49-year-old right-handed female with an $\mathrm{ICH}$ in the left corona radiata and basal ganglia, who underwent conservative treatment at the neurosurgery department of a university hospital. Brain CT performed at one week after onset showed an ICH in the left corona radiata and basal ganglia, and a left-to-right midline shift of $6.5 \mathrm{~mm}$ (Fig. 1A). Brain MRI performed at one month after onset showed a leukomalatic lesion in the same regions (Fig. 1A). On K-WAB performed at one and three months after onset, the patient presented with Broca's apha-

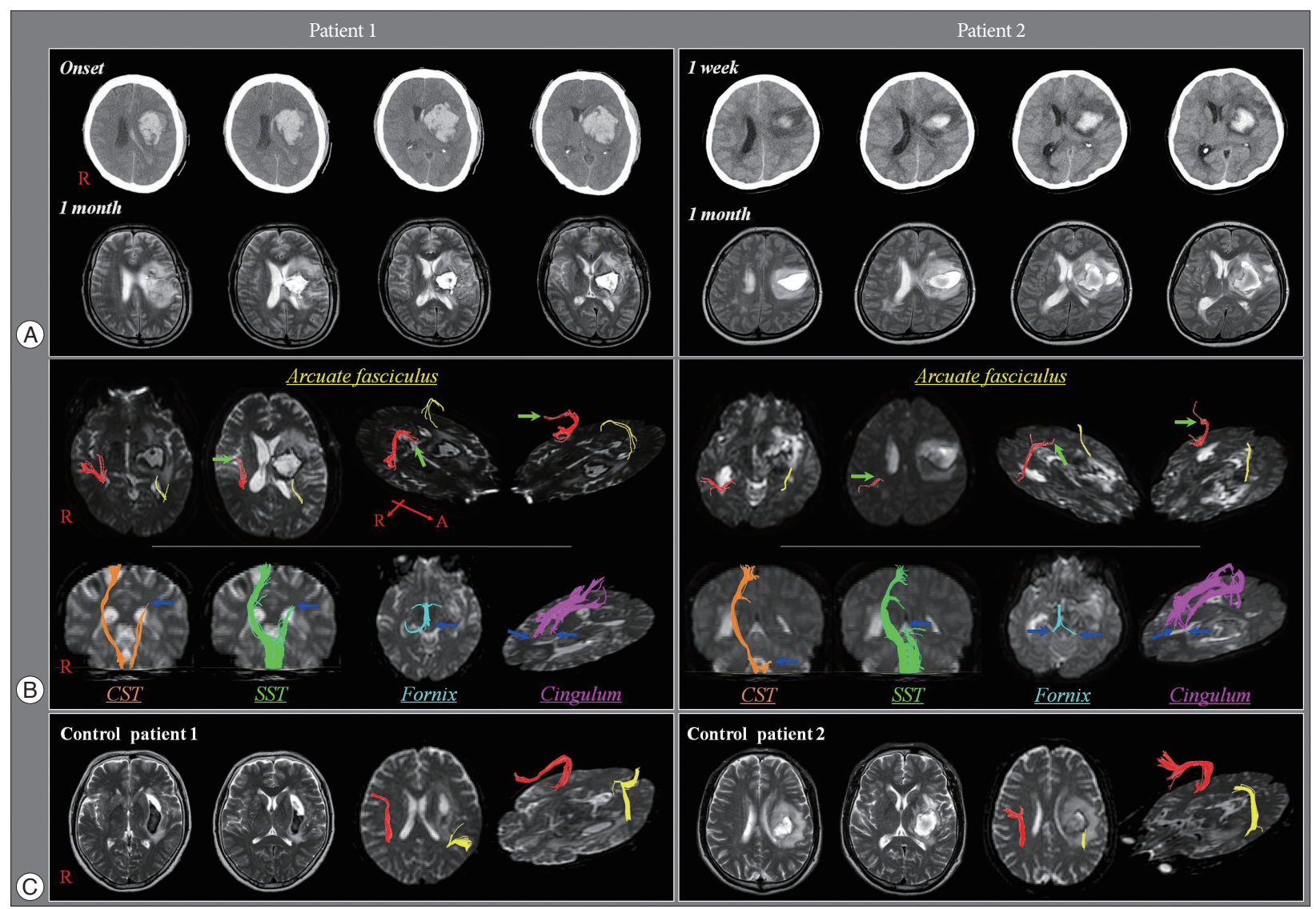

Fig. 1. A : Brain computed tomography images show an intracranial hemorrhage in the left corona radiata and basal ganglia and a left-to-right midline shift. T-2 weighted brain images show a leukomalatic lesion in the left corona radiata and basal ganglia. B : Diffusion tensor tractography (DTT) for the patient 1 and patient 2 shows disruptions of the arcuate fasciculus (AF) in both hemispheres. The right arcuate fasciculus in patient 1 and patient 2 are disrupted around the middle and posterior portions of the horizontal portion, respectively (arrow). The left arcuate fasciculus in both patients is disrupted at the junction between the ascending and horizontal portions. C : DTT for the control patients showed the preservation of the right AFs and the discontinuation of the left AFs after originating from Wernicke area. 
sia. The aphasia showed improvement at three months after onset, compared with that at one month after onset (one monthaphasia quotient : 15 , fluency: 5.8 , comprehension : 72 , repetition : 0 , naming : 13 ; three months - aphasia quotient : 28 , fluency $: 6$, comprehension : 137 , repetition : 14.5 , naming : 26.3 ).

\section{Diffusion tensor tractography}

DTI was performed at one month after onset in both patients using a sensitivity-encoding head coil on a 1.5-T Philips Gyroscan Intera (Hoffman-LaRoche Ltd., Best, The Netherlands) with single-shot echo-planar imaging and navigator echo. Sixty contiguous slices (acquisition matrix $=96 \times 96$; reconstruction matrix $=192 \times 192$ matrix; field of view $=240 \times 240 \mathrm{~mm}^{2} ; \mathrm{TR}=10726$ $\mathrm{ms}$; TE=76 ms, $\mathrm{b}=1,000 \mathrm{~s} / \mathrm{mm}^{2}, \mathrm{NEX}=1$, thickness $=2.5 \mathrm{~mm}$ ) were acquired for each of the 32 noncollinear diffusion-sensitizing gradients. Fiber tracking was performed using the fiber assignment continuous tracking (FACT) algorithm implemented within the DTI task card software (Philips Extended MR WorkSpace 2.6.3). Removal of eddy current-induced image distortions using affine multi-scale two-dimensional registration was performed at the Oxford Centre for Functional Magnetic Resonance Imaging of Brain (FMRIB) Software Library (FSL; www.fmrib.ox.ac.uk/fsl). DTI-Studio software (CMRM, Johns Hopkins Medical Institute, Baltimore, MD, USA) was used for evaluation of the AF. Based on the method of Nucifora et al. ${ }^{15)}$, we placed the region of interest (ROI) for tracking of the AF (the seed ROI in the posterior parietal area of the superior longitudinal fascicle and the target ROI in the posterior temporal lobe). The seed ROI was shown in green and the target ROI was blue on DTI-based color-coded maps. Termination criteria used for fiber tracking was fractional anisotropy (FA) $<0.2$ and angle $<60^{\circ}$. We also measured the fractional anisotropy (FA) and apparent diffusion coefficient (ADC) values and the tract number of the AF. Also, we evaluated corticospinal tract (CST), spinothalamic tract (STT), fornix, and cingulum. For placing ROIs, we applied the methods which were used in our previous study ${ }^{13)}$.

The right AFs of patient 1 and patient 2 were disrupted between Wernicke's and Broca's areas. The ascending portions from Wernicke's area were spared, however, the remaining portions (i.e., horizontal portion) of the right AFs were disrupted around the middle (patient 1) (Fig. 1B) and posterior portions (patient 2) (Fig. 1B), respectively. In addition, the left AFs of patient 1 and patient 2 were disrupted at the junction between the ascending and horizontal portions (around the brain lesions) (Fig. 1B), and horizontal portions of the left AFs were not reconstructed. In contrast, in all control patients, whereas disruptions of the left AFs were found between Wernicke's and Broca's areas, the right AFs originating from Wernicke's area passed through the known AF pathway and then terminated in Broca's area (Fig. 1C). The FA value and tract numbers of the right AFs in patient 1 and patient 2 were more than two standard deviations lower than those of control patients (Table 1). In contrast, the ADC value was more than two standard deviations higher than that of con-
Table 1. Diffusion tensor tractography parameters for the arcuate fasciculus in ICH patients with subfacine herniation and control patients without subfacine herniation

\begin{tabular}{lcc}
\hline & Right & Left \\
\hline Patient 1 & & \\
FA & $0.406^{*}$ & 0.337 \\
ADC & $0.878^{\dagger}$ & 1.066 \\
Tract number & $187^{\star}$ & 49 \\
Patient 2 & & \\
FA & $0.385^{\star}$ & 0.378 \\
ADC & $0.875^{\dagger}$ & 0.890 \\
Tract number & $126^{\star}$ & 111 \\
Control patients & & \\
FA & $0.482(0.019)$ & $0.342(0.033)$ \\
ADC & $0.765(0.031)$ & $0.982(0.047)$ \\
Tract number & $1243(87)$ & $101(30)$ \\
\hline
\end{tabular}

Control data are presented as mean ( \pm standard deviation). *More than two standard deviations lower than that of normal control values, ${ }^{\dagger}$ More than two standard deviations higher than that of normal control values. ICH : intracerebral hemorrhage, FA : fractional anisotropy, ADC : apparent diffusion coefficient

trol patients (Table 1).

As for other neural tracts (CST, STT, fornix, and cingulum), all the evaluated neural tracts on the left hemisphere were disrupted by the brain lesions in patient 1 and patient 2 . On the other hands, the integrities of CST and STT on the right hemisphere were preserved in patient 1 and patient 2 , and fornix on the right hemisphere was also preserved in patient 1 . However, the anterior portion of the right cingulum was disrupted in patient 1 and patient 2, and the right crus of fornix was disrupted in patient 2 .

In the comparison of the severity of aphasia between ICH patients with subfacine herniation and control patients, the aphasia quotient at three months after onset in patient 1 (39) and patient 2 (28) were more than two standard deviations lower than those of control patients (aphasia quotient at three months : $57.3 \pm 8.3$, mean \pm standard deviation).

\section{DISCUSSION}

In the current study, using the configuration and parameters of DTT, we investigated injury of the AF in the nondominant (right) hemisphere as well as that in the dominant (left) hemisphere in two patients with subfalcine herniation following the $\mathrm{ICH}$ in the dominant hemisphere. On DTT, the right AFs of both patients showed disruption between Wernicke's and Broca's areas, although conventional CT and MRI did not show any remarkable lesions in the right hemisphere. The integrity of the ascending portions of the right AF was preserved, however, the other portion of the AF did not reach Broca's area. The left AFs in both patients were disrupted after originating from Wernike's area. These DTT findings for the AFs in the dominant hemisphere appear to show correlation with Broca's aphasia, which was presented in both patients. In addition, the FA values and 
tract numbers for the right AFs showed a significant decrease in both patients, compared with normal subjects, whereas the $\mathrm{ADC}$ values of the right AFs showed a significant increase. The decrease in FA value resulted from deterioration of directional microstructures, such as axon, myelin, and microtubules ${ }^{2,14)}$. In contrast, the increase in ADC value may indicate some pathology, such as vasogenic edema or accumulation of cellular debris from axonal damage ${ }^{2,14)}$. Deterioration of neuronal microstructure in a neural tract can result in reduction of tract numbers. These results for DTT parameters (decrease of FA value and tract number and increase of $\mathrm{ADC}$ value) in our patients indicate damage of the AFs in the right hemispheres.

It is believed that the left AF in both patients injured by the left $\mathrm{ICH}$ because the left AF was disrupted around the hematoma. Regarding injury to the right $\mathrm{AF}$, the most plausible cause of that injury seems to be the subfalcine herniation. We think that the left-to-right subfalcine herniation induced the rightward compressing force to the right cerebral hemisphere, which consequently resulted in compression or injury of the right AF. Further, we can also suppose that increased intracranial pressure induced by subfalcine herniation leads to decreased cerebral perfusion of the right hemisphere ${ }^{17)}$, which might cause ischemic injury to the right AF.

The current study is the first study to demonstrate injury of the $\mathrm{AF}$ in the nondominant hemisphere by subfalcine herniation after ICH, using DTT. Many previous studies have demonstrated that the nondominant hemisphere areas homologous to the dominant hemisphere language areas (Broca's and Wernicke's areas) and the AF connecting those areas have a significant role in recovery of language dysfunction. ${ }^{7,8)}$ It is assumed that the prognosis for aphasia in patients with damage to both AFs would be worse than that in patients with AF damage in only the dominant hemisphere. The worse language function of the patients in this study appears to be related with injury of the AF in the nondominant hemisphere. Accordingly, evaluation of the AF in the nondominant hemisphere using DTT would be necessary in patients with subfalcine herniation due to left brain lesions. Additionally, although AFs on both hemispheres were disrupted in patient 1 and patient 2 , aphasia at three months after onset was improved, compared with that at one month after onset. We can assume the recovery of aphasia in these patients occurred via other neural tracts such as the superior longitudinal fasciculus ${ }^{12)}$.

\section{CONCLUSION}

Using the configuration and parameters of DTT, we confirmed injury of the AF in the nondominant hemisphere in two patients with subfalcine herniation following ICH in the dominant hemisphere, although no lesion was observed in the nondominant hemisphere on conventional CT and MRI. Therefore, DTT would be a useful tool for detection of underlying injury of the AF in patients with subfalcine herniation. However, this study is limited in that it is a case report. Conduct of additional studies involving larger case numbers of patients with subfalcine herniation is warranted.

\section{- Acknowledgements}

This work was supported by the DGIST R\&D Program of the Ministry of Science, ICT and Future Planning (16-BD-0401).

\section{References}

1. Adams RD, Victor M, Ropper AH : Principles of neurology, ed 6. New York : McGraw-Hill, 1997

2. Assaf Y, Pasternak O : Diffusion tensor imaging (DTI)-based white matter mapping in brain research : a review. J Mol Neurosci 34 : 51-61, 2008

3. Bartha L, Benke $\mathrm{T}$ : Acute conduction aphasia : an analysis of 20 cases. Brain Lang 85 : 93-108, 2003

4. Byard RW : Patterns of cerebral and cerebellar herniation. Forensic Sci Med Pathol 9: 260-264, 2013

5. Catani M, Mesulam $\mathrm{M}$ : The arcuate fasciculus and the disconnection theme in language and aphasia : history and current state. Cortex 44 : 953-961, 2008

6. Duffau H, Capelle L, Sichez N, Denvil D, Lopes M, Sichez JP, et al. : Intraoperative mapping of the subcortical language pathways using direct stimulations. An anatomo-functional study. Brain 125 (Pt 1) : 199-214, 2002

7. Heiss WD, Kessler J, Thiel A, Ghaemi M, Karbe H : Differential capacity of left and right hemispheric areas for compensation of poststroke aphasia. Ann Neurol 45 : 430-438, 1999

8. Heiss WD, Thiel A : A proposed regional hierarchy in recovery of poststroke aphasia. Brain Lang 98 : 118-123, 2006

9. Hong JH, Kim SH, Kim OL, Byun WM, Jang SH : Neural tract injuries by brain herniations after head trauma. J Head Trauma Rehabil 27 : 154-158, 2012

10. Hosomi A, Nagakane Y, Yamada K, Kuriyama N, Mizuno T, Nishimura T, et al. : Assessment of arcuate fasciculus with diffusion-tensor tractography may predict the prognosis of aphasia in patients with left middle cerebral artery infarcts. Neuroradiology 51 : 549-555, 2009

11. Kim H, Na DL : Normative data on the Korean version of the Western Aphasia Battery. J Clin Exp Neuropsychol 26 : 1011-1020, 2004

12. Kim SH, Jang SH: Prediction of aphasia outcome using diffusion tensor tractography for arcuate fasciculus in stroke. AJNR Am J Neuroradiol 34 : 785-790, 2013

13. Lee AY, Shin DG, Park JS, Hong GR, Chang PH, Seo JP, et al. : Neural tracts injuries in patients with hypoxic ischemic brain injury : diffusion tensor imaging study. Neurosci Lett 528 : 16-21, 2012

14. Neil JJ : Diffusion imaging concepts for clinicians. J Magn Reson Imaging $27: 1-7,2008$

15. Nucifora PG, Verma R, Melhem ER, Gur RE, Gur RC : Leftward asymmetry in relative fiber density of the arcuate fasciculus. Neuroreport 16 : 791-794, 2005

16. Payabvash S, Kamalian S, Fung S, Wang Y, Passanese J, Kamalian S, et al. : Predicting language improvement in acute stroke patients presenting with aphasia : a multivariate logistic model using location-weighted atlas-based analysis of admission CT perfusion scans. AJNR Am J Neuroradiol 31 : 1661-1668, 2010

17. Rosner MJ, Rosner SD, Johnson AH : Cerebral perfusion pressure : management protocol and clinical results. J Neurosurg 83 : 949-962, 1995

18. Thulborn KR, Carpenter PA, Just MA : Plasticity of language-related brain function during recovery from stroke. Stroke 30 : 749-754, 1999 